Ann. rheum. Dis. (1979), 38, Supplement p. 52

\title{
Sexually acquired reactive arthritis ${ }^{1}$
}

\author{
A. C. KEAT, J. T. SCOTT, G. RIDGWAY, R. N. MAINI, \\ AND G. D. PEGRUM \\ From the Charing Cross Hospital and University College Hospital, London
}

Arthritis is a widely seen complication of genitourinary infection (especially in the United Kingdom and United States). Although a genetic predisposition-presence of HLA-B27-is well recognised diagnostic criteria are inadequate. Both the nature of the causal infective organism and the pathogenetic mechanisms are unknown.

\section{Patients}

We have recently published the findings of a prospective study of 531 men with non-gonococcal urethritis with particular reference to the frequency of isolation of Chlamydia trachomatis and the presence of HLA-B27.175 We now present more detailed findings in 57 patients (some of them taken from the prospective study) with arthritis consequent upon non-gonococcal genital infectionsexually acquired reactive arthritis (SARA) - with regard to the clinical form of the disease, the possible role of $C$. trachomatis as a trigger agent, and the likelihood of direct interaction between this and HLA-B27. Thirty-one of the patients presented themselves in the first place to a rheumatology clinic and 26 to a department of genitourinary medicine.

\section{Clinical features}

The most common clinical presentations were knee effusion or painful swelling of an ankle or metatarsophalangeal joint occurring on average 14 days after the onset of urethritis. So far as calculation is possible, the mean incubation period for the development of rheumatic disease was 28 days. Nearly half the patients presented with monarthritis, but fasciitis and tenosynovitis were also seen as initial features. Joint disease was almost invariably an asymmetrical oligoarthritis affecting principally the knees, ankles, and metatarsophalangeal joints.

Spinal and sacroiliac arthritis were not prominent features. Early sacroiliitis was diagnosed on clinical or radiological grounds in five patients. Inflammatory

\footnotetext{
${ }^{1}$ This paper was presented by Dr. J. T. Scott.
}

Table 1 Clinical features in 57 patients with sexually acquired reactive arthritis $(S A R A)$

\begin{tabular}{lll}
\hline Sex ratio (M:F) & $10: 1$ \\
Incubation period & 28 days \\
Onset & Acute oligo/monarticular \\
Peripheral joint disease & \multicolumn{2}{c}{ Asymmetrical oligoarthritis } \\
Sacroiliitis & 5 patients & $(9 \%)$ \\
Spondylitis & 1 patient & $(2 \%)$ \\
Enthesopathy & 19 patients & $(33 \%)$ \\
Tenosynovitis & 12 patients & $(21 \%)$ \\
Mucocutaneous lesions & 11 patients & $(19 \%)$ \\
Conjunctivitis & 12 patients & $(21 \%)$ \\
Iritis & 3 patients & $(5 \%)$ \\
Classical Reiter's syndrome & 15 patients & $(23 \%)$ \\
\hline
\end{tabular}

foci at tendinous or fascial entheses were character istic, as in ankylosing spondylitis. They occurred as tender swellings, usually over the calcaneum at the? insertion of the Achilles tendon or plantar fascia but also over the lower ribs, around the tibia at the knees, around the pelvis, and at the insertion of erector spinae fibres at the occiput. Tenosynovitis affected particularly the dorsal tendons of the foot and hand. Ocular and mucocutaneous lesions, as classically described in Reiter's syndrome, were each seen in only about one-fifth of the patients (Table 1).

\section{HLA-B27}

Out of 54 patients tissue-typed $36(67 \%)$ were B27positive. Whereas 25 of the 30 tissue-typed patients $(83 \%)$ referred directly to the rheumatology unit possessed the antigen, only 11 of 24 patients $(46 \%)$ presenting at a venereal disease clinic were B27positive. The figures compare with an antigen prevalence of $6 \%$ in uncomplicated non-gonococcal urethritis and $7 \%$ in 280 healthy controls. The difference in prevalence of B27 positivity found between the rheumatology and genitourinary clinics probably reflects severity of joint disease. Patients lacking the antigen had fewer joints involved, a shorter duration of the acute joint episode, and a reduced chance of extra-articular lesions or progress to chronic disease (Table 2). 
Table 2 Disease features in $H L A-B 27$-positive and HLA-B27-negative patients

\begin{tabular}{lll}
\hline & \multicolumn{2}{l}{$H L A-B 27$} \\
\cline { 2 - 3 } & Positive & Negative \\
\hline Number of joints affected & $3 \cdot 0$ & $2 \cdot 2$ \\
Soft tissue lesions & $58 \%$ & $28 \%$ \\
Mucocutaneous lesions & $25 \%$ & $6 \%$ \\
Iritis & $8 \%$ & 0 \\
Episode duration (mean) & 24.9 weeks & $10 \cdot 6$ weeks \\
Chronic course (1 year) & $14 \%$ & $6 \%$ \\
\hline
\end{tabular}

\section{Reactive arthritis}

In many aspects the rheumatic disease we have described resembles the 'reactive arthritis' which occurs after salmonella, shigella, or yersinia infections of the bowel (although in these conditions mucocutaneous manifestations are uncommon and the sex ratio approximates to one). Identical disease features are seen in other patients, many of them possessing the antigen HLA-B27, but in whom no evidence of causal infection can be established. Thus at present there appear to be three broad categories of reactive arthritis: (a) sexually acquired, (b) enterocolitic, and (c) idiopathic or of unknown aetiology.

Suggested criteria for SARA are set out in Table 3. A diagnosis of definite SARA would be met by the presence of all essential criteria with at least two musculoskeletal criteria, or one musculoskeletal and one systemic criterion. A diagnosis of probable SARA would be met by the presence of all essential criteria with one musculoskeletal criterion. These suggested criteria have not been formally tested.

\section{Chlamydia trachomatis as trigger factor}

Chlamydia trachomatis infection is found in up to $60 \%$ of men with uncomplicated non-gonococcal urethritis and is thought to play a causal role. In a

Table 3 Sexually acquired reactive arthritis

Essential criteria
(1) Recent sexual contact
(2) Definite non-gonococcal urethritis or mixed infection
(3) Absent IgM rheumatoid factor
(4) Exclusion of metabolic or infective lesions
Clinical criteria
(a) Musculoskeletal
(1) Characteristic arthritis
(2) Enthesopathy
(3) Tenosynovitis
(b) Systemic
(1) Ocular inflammation
(2) Mucocutaneous lesions
(3) Raised ESR
(4) HLA B27-positive

selective prospective study of men presenting with non-gonococcal urethritis cultures were obtained from 491, 19 of whom developed reactive arthritis. Positive isolates were obtained from about one-third of the patients independently of the occurrence of arthritis (Table 4), indicating that $C$. trachomatis is not an exclusive trigger factor for sexually acquired reactive arthritis. However, antibody studies currently being carried out in conjunction with Drs Brenda Thomas and David Taylor-Robinson at the MRC Clinical Research Centre, London, suggest that the incidence of $C$. trachomatis may be considerably higher in both groups and, furthermore, that the magnitude of the immune response is greater in patients with reactive arthritis than in those with uncomplicated urethritis. Details of this work, now in preparation, will shortly be published elsewhere.

In order to explore the possibility that the presence of HLA-B27 could predispose to SARA by rendering the patient more likely to develop chlamydial infection, the incidence of chlamydial isolates was assessed in B27-positive and B27-negative patients with reactive arthritis and uncomplicated non-gonococcal urethritis (Table 5). The isolation rate was similar in all four groups.

\section{Conclusions}

We suggest that the generic term 'reactive arthritis' should apply to a broad group of diseases (including Reiter's syndrome, which term in itself is of little value) with three categories: (a) sexually acquired (SARA), (b) enterocolitic, and (c) idiopathic or of

Table 4 Urethral cultures for $\mathrm{C}$. trachomatis in sexually acquired reactive arthritis $(S A R A)$ and nongonococcal urethritis (NGU)

\begin{tabular}{lccl}
\hline & No. & Isolate & \\
\cline { 3 - 4 } & & Positive & $\%$ Positive \\
\hline SARA & 19 & 7 & $36 \cdot 8$ \\
NGU & 472 & 167 & 35.4 \\
\hline
\end{tabular}

Table 5 Isolation of $\mathrm{C}$. trachomatis in relation to HLA-B27 status

\begin{tabular}{|c|c|c|c|c|}
\hline & \multicolumn{2}{|l|}{$N G U$} & \multicolumn{2}{|l|}{$S A R A$} \\
\hline & $\begin{array}{l}\text { B27- } \\
\text { positive }\end{array}$ & $\begin{array}{l}\text { B27- } \\
\text { negative }\end{array}$ & $\begin{array}{l}\text { B27- } \\
\text { positive }\end{array}$ & $\begin{array}{l}\text { B27- } \\
\text { negative }\end{array}$ \\
\hline $\begin{array}{l}\text { Total no. } \\
\text { Isolate positive }\end{array}$ & $\begin{array}{r}15 \\
7\end{array}$ & $\begin{array}{l}88 \\
43\end{array}$ & $\begin{array}{l}8 \\
3\end{array}$ & $\begin{array}{r}11 \\
4\end{array}$ \\
\hline$\%$ Isolate positive & 47 & 49 & 38 & 36 \\
\hline
\end{tabular}


unknown cause. HLA-B27 is present in $67 \%$ of patients with SARA. Patients possessing the antigen develop more severe disease than those without. C. trachomatis infection is found in some but not all patients with SARA and cannot therefore be an exclusive trigger factor, though a possible role in a proportion of cases cannot be excluded. HLA-B27 does not influence susceptibility to genital infection by $C$. trachomatis.

\section{General discussion}

DR. D. A. BREWERTON: I congratulate the authors on their title. All day long we have been tripping over what goes into the box called 'Reiter's disease' and they have had the courage to break right away from it and say what they mean. I particularly enjoyed the slide showing that reactive arthritis was either sexually acquired, or related to intestinal infection, or of unknown cause. I think that is much more honest than some other approaches to the problem.

DR. A. CALIN: I seem to remember, Dr. Scott, from your previous paper that $20 \%$ of the B27-positive patients who happened to have chlamydial infection developed Reiter's - I am sorry, reactive arthropathy.

DR. SCOTT: No, it was $20 \%$ (six of out 30) B27positive patients with non-gonococcal urethritis, not necessarily with chlamydial infection, who developed reactive arthritis. The incidence of chlamydia in the B27-positive patients with a satisfactory culture was not different from that in the whole series, and there was no significant difference in the incidence of arthritis in the culture-positive and culture-negative groups.

DR. CALIN: Is it a coincidence that the $20 \%$ figure has come up in many other populations-for example, $20 \%$ of healthy B27-positive people developing AS, the salmonella studies of Aho, and so on?

DR. SCOTT: You think it might just be a coincidence? DR. CALIN: Either that or $20 \%$ of B27-positive individuals have a certain genetic make-up.

DR. SCOTT: It seems that about $20 \%$ of B27-positive subjects who develop urogenital or gut infection develop reactive arthritis, but $I$ am not sure how this relates to $20 \%$ of a global B27-positive population developing ankylosing spondylitis.

PROF. E. S. PERKINS: I have just talked to our venereologist, Dr. Dunlop, about the isolation of chlamydia. His figures correspond extremely well with those that you have given. He said that he was getting about $40 \%$ of isolates in non-specific urethritis without complications and the same proportion in those cases with reactive arthritis. There is no relationship to $\mathrm{B} 27$.

DR. CSONKA: I was interested in Dr. Scott's figures and those reported from Dr. Dunlop. In the Middle East, where I have been recently, we found chlamydia positive in about $30 \%-40 \%$ of patients with NSU and about the same number in patients who did not have NSU, which is rather upsetting, and incidentally in $30 \%-40 \%$ of patients with gonorrhea. Now this has also been found in Bristol by Dr. Richmond, so I wonder how important is chlamydia?

DR. SCOTT: These were really healthy people?

DR. CSONKA: Yes, completely healthy people, medical or technicians. They had no trachoma.

DR. SCOTT: It would be interesting to hear about antibody studies in these groups.

DR. CSONKA: They were done, and showed no? difference.

PROF. R. F. WILLKENS: In our area Dr. David Martin, working with Dr. King Holmes, is doing a lymphocyte stimulation test with chlamydia, testing the lymphocytes of patients with Reiter's syndrome. $\mathrm{He}$ is finding that about one half of his patients are stimulation-positive and one half stimulationnegative.

DR. SCOTT: I think that chlamydial infection is probably important in a proportion of cases of nongonococcal urethritis, but its eaxct role is subsequent reactive arthritis is uncertain. The incidence of chlamydial isolates is about the same in uncompleted non-gonococcal urethritis as in SARA, but it looks as if antibody titres are higher in the reactive arthritis patients, suggesting some sort of pathogenic mechanism. 